\title{
“活”的 $\alpha$-甲基苯乙烯共聚物：聚合反应新化学和材料工程新技术
}

\author{
马育红 ${ }^{a} \quad$ 张冰 $^{a} \quad$ 赵长稳 ${ }^{a} \quad$ 刘莲英 ${ }^{a}$ 蒋姗 $^{b}$ \\ 梁淑君 ${ }^{c} \quad$ 杨万泰*, ${ }^{*}$ \\ ( ${ }^{a}$ 北京化工大学碳纤维及功能高分子教育部重点实验室 化工资源有效利用国家重点实验室 北京 100029)

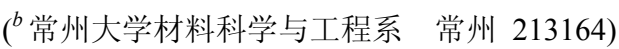 \\ $\left({ }^{c}\right.$ 太原工业学院材料工程系 太原 030008)
}

\begin{abstract}
摘要 高于临界聚合反应温度时, $\alpha$-甲基苯乙烯(AMS)单体和其聚合物处于聚合-解聚平衡. 基于 AMS 聚合物在受热时 可裂解生成大分子链自由基的特性，提出了含 AMS 结构单元的共聚物是一种 “活” 的，可作为大分子自由基引发剂的 概念, 并通过实验对 AMS 共聚物的引发性能和应用进行了研究. 首先, 合成了 AMS 与(甲基)丙烯酸酯类单体、丙烯酸、 苯乙烯和马来酸䣷等的共聚物. 然后以上述共聚物为大分子引发剂, 在 $90{ }^{\circ} \mathrm{C}$ 引发(甲基)丙烯酸酯类单体和苯乙烯等的 本体聚合、溶液聚合和乳液聚合, 得到了嵌段共聚物. 用 ESR 谱证明了 AMS 的共聚物在加热时能裂解生成以碳原子 为中心的大分子链自由基. 此外, 在聚合物的熔融共混中, AMS 分解产生的大分子链自由基通过偶合反应形成接枝链, 原位生成相容剂. AMS 共聚物还可以对碳纳米管及无机粒子进行表面原位接枝改性. AMS 共聚物是一类无小分子残留 的绿色自由基引发剂，可以用于低成本制备两嵌段共聚物，也可以用于聚合物的熔融共混增容.
\end{abstract}

关键词 自由基聚合; 大分子引发剂; $\alpha$-甲基苯乙烯; 嵌段聚合物; 接枝聚合; 反应性增容

\section{A Novel Macroinitiator Based on the Copolymer of $\alpha$-Methylstyrene Synthesis and Its Application in Preparing Block and Graft Polymers}

\author{
Ma, Yuhong ${ }^{a}$ \\ Zhang, Bing ${ }^{a}$ \\ Zhao, Changwen ${ }^{a}$ \\ Liu, Lianying ${ }^{a}$ \\ Jiang, Shan ${ }^{b}$ \\ Liang, Shujun ${ }^{c} \quad$ Yang, Wantai*,a \\ ( ${ }^{a}$ Key Laboratory of Carbon Fiber and Functional Polymers, Ministry of Education, \\ Beijing University of Chemical Technology, Beijing 100029, China) \\ ( ${ }^{b}$ Department of Materials Science and Engineering, Changzhou University, Changzhou 213164, China) \\ ( ${ }^{c}$ Department of Materials and Engineering, Taiyuan Institute of Technology, Taiyuan 030008, China)
}

\begin{abstract}
Due to the steric hindrance effects in combination with stability of the tertiary benzylic $\alpha$-methylstyryl radical, there is a dynamic equilibrium between the monomer $\alpha$-methylstyrene (AMS) and its polymer (PAMS) when the temperature is greater than $61{ }^{\circ} \mathrm{C}$ (the ceiling polymerization temperature). Based on this unique feature, a novel strategy to prepare copolymers of AMS having liable bonds as potential macromolecular free radical initiators for synthesizing block and graft copolymers has been successfully developed in our laboratory. By conventional free radical polymerization, a series of AMS copolymers, including copolymers with (meth)acrylate, acrylic acid, styrene and maleic anhydride were synthesized. Typically, with the increasing of AMS fraction in monomer feed, the rate of copolymerization was significantly retarded and the molecular weight of the copolymers was reduced. However, the copolymer yield could be as high as $90 \%(w)$ with the increased addition of initiator, up to $4 \%(w)$, and the molar fraction of AMS structural unit in AMS copolymers could be up to $25 \%(\mathrm{~mol} \%)$. It has been demonstrated that the copolymers containing AMS structural units are efficient free radical initiators when the temperature is greater than $80{ }^{\circ} \mathrm{C}$ (much better higher than $90{ }^{\circ} \mathrm{C}$ ). These copolymers could be exploited as macroinitiator in preparing block copolymers and core-shell polymer particles by bulk, solution and emulsion polymerization processes. In addition, the experimental results demonstrated that the molecular weight of copolymer products prepared with AMS copolymers as macromolecular initiators increased steadily with the monomer conversion. Though the polymerization initiated by AMS copolymers was not a well-controlled living system yet, it showed some characteristics of living polymerization. The ESR spectrum presented direct evidence of the generation of carbon centered radicals in the products of copolymer of AMS with glycidyl methacrylate (PAG) heated with $N$ - $t$-butyl- $\alpha$-phenylnitrone at $90{ }^{\circ} \mathrm{C}$ in toluene. Besides initiating the polymerization of vinyl monomer to prepare diblock copolymer, the AMS copolymers offered a practical pathway to synthesize grafting polymers in melting state. For example, with the addition of PAG in the PP/Nylon melten blending, it has been demonstrated a significant in situ compatibilization effect and the formation of graft polymer of PAG and PP. Furthermore, the AMS copolymers could also be used to modify MWCNT by free radical grafting onto mechanism. Instead of com-
\end{abstract}

*E-mail: yangwt@mail.buct.edu.cn

Received October 31, 2012; published December 18, 2012.

Project supported by the National Natural Science Foundation of China (No. 51221002-E03).

项目受国家自然科学基金委员会创新研究群体科学基金(No. 51221002-E03)资助. 
peting with the other existing controlled free radical polymerization technologies, the AMS copolymer method, with competitive cost and no small molecular residues, offers an alternative tool for polymer chemists to develop block copolymers on an industrial scale for some applications, such as dispersant and compatibilizer.

Keywords free radical polymerization; macroinitiator; $\alpha$-methylstyrene; block copolymer; grafting polymerization; reactive compatibilization

\section{1 引言}

由于适用单体范围广、实施方法多和成本低, 目前 仍有大约 50\%的乙烯基聚合物是按传统自由基聚合方 法生产的 ${ }^{[1]}$. 其聚合机理主要特点是, 慢引发 $\left(k_{d}<10^{-5}\right.$ $\left.\mathrm{s}^{-1}\right)$, 快增长 $\left(k_{p} \approx 10^{3} \mathrm{~mol}^{-1} \cdot \mathrm{L} \cdot \mathrm{s}^{-1}\right)$, 易终止 $\left(k_{t} \approx 10^{7}\right.$ $\left.\mathrm{mol}^{-1} \cdot \mathrm{L} \cdot \mathrm{s}^{-1}\right)$ 和链转移 $[2,3]$; 导致难以对聚合产物的分子 结构、相对分子质量及其分布进行设计和调控.

1982 年, Otsu 等 ${ }^{[4,5]}$ 用难以引发单体聚合的小分子 稳定自由基调控聚合反应，提出了实现 “活性” 自由基

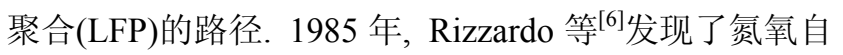
由基调控的苯乙烯 LFP. 1995 年, Matyjaszewski 等 ${ }^{[7]}$ 的 ATRP 体系, Sawamoto 等 ${ }^{[8]}$ 的过渡金属催化体系, 以及 Percec 等 ${ }^{[9]}$ 的芳磺酰氯 $/ \mathrm{Cu}(\mathrm{bpy}){ }_{n} \mathrm{Cl}$ 体系成为 LFP 获得突 破的标志. 三年后, CSICO 实验室 ${ }^{[10]}$ 报道了另一类重要 的 RAFT 体系. 上述 LFP 都存在一个休眠种 C-X (X) $\mathrm{S}, \mathrm{Mt}$ )和活性增长链自由基间的可逆动态平衡. 由于这 些体系本身的缺陷, 如铜残留, 颜色/味道等, 迄今仍难 应用于大宗聚合物品种生产.

1,1 二苯基乙烯(DPE), 历史上曾被应用于碳负离 子 $^{[11]}$ 或碳正离子 ${ }^{[12]}$ 控制聚合体系, 通过其对活性中心 加成来降低活性种的反应性, 进而再引发新单体的聚 合、对聚合物链进行官能化和对活性种进行转化. 2001 年 Nuyken 等 ${ }^{[13]}$ 报道, 加入 DPE 于传统自由基聚合体系 后, 体系表现出可控聚合的特征. DPE 与链增长自由基 $P_{n}{ }^{*}$ 加成, 生成低反应活性的链自由基 $P_{n}-D^{*}$, 聚合反应 速率和产物的 $\overline{M_{\mathrm{w}}} / \overline{M_{\mathrm{n}}}$ 明显降低, 而且这一反应是可逆
的. 此外, 两个 $P_{n}-D^{*}$ 还可以偶合生成半醌式结构, 偶合 产物在受热时能重新分解成自由基(图式 1$)^{[14,15]}$. 加入 新单体，可用于制备嵌段共聚物，如 PMMA- $b-\mathrm{PS}^{[13]}$, PMMA- $b-$ PBA $^{[15]}$ 和 PMMA- $b-P V A c^{[16]}$, 以及核壳粒子 等 ${ }^{[17,18]}$. 与其它的 LFP 相比, DPE 体系的优点是无须使 用金属催化剂和/或杂原子链转移剂; 缺点是, DPE 是非 聚合单体, 在聚合过程中只能作为封端剂, 一个大分子 链最多只能有一个潜在的活性点, 产物相对分子质量分 布较宽，嵌段聚合时存在一定量的均聚物，因此应用受 到了一些限制, 如只能用于特殊分散剂和相容剂等的制 备 ${ }^{[19]}$.

在高分子化学教科书中, $\alpha$-甲基苯乙烯(AMS)是最 典型的热力学不聚合或难聚合单体, 当反应温度大于 $61{ }^{\circ} \mathrm{C}$ 时, 其自由基聚合处于聚合一解聚平衡 ${ }^{[20,21]}$. 但是, 利用特殊手段(低温阴离子或阳离子聚合)得到的 AMS 均聚物却具有很好的耐热性 $\left(T_{\mathrm{g}}\right.$ 高达 $\left.168{ }^{\circ} \mathrm{C}\right)$. 并且, 当 AMS 单元经共聚引进苯乙烯类聚合物中时, 不仅可提 高力学性能, 还可提高共聚物的 $T_{\mathrm{g}}$, 热稳定性和辐射稳 定性. 对上述结果和现象深入思考后, 我们认为: 当温 度高于该聚合物或共聚物的解聚温度时, 其解聚实际上 是一个链断裂产生链自由基的过程, 但断裂产生的链自 由基夺氢能力弱 ${ }^{[22]}$, 易重新偶合形成共价键，由此形成 一个动态的分子链裂解成自由基-自由基偶合重新生成 大分子链的平衡, 使得聚合物或共聚物仍有高的稳定 性. 此时, 体系中若有其它链自由基或单体存在, PAMS 裂解生成的自由基可以进行其它反应，例如通过偶合反 应引入不可逆单元(接枝或嵌段)或引发单体聚合(嵌段<smiles>C=CCCCC(C)(C)CC(C=C)(c1ccccc1)c1ccccc1</smiles>

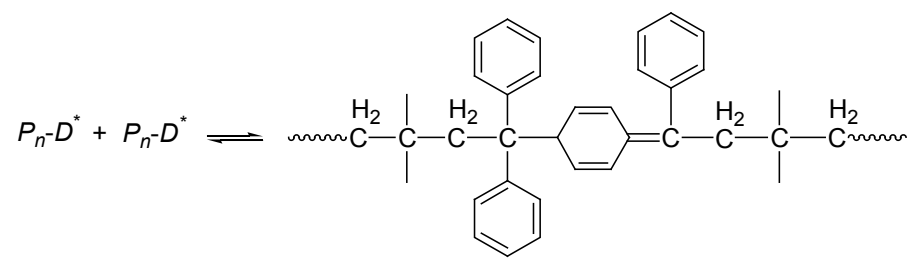

(B)

图式 1 DPE 控制聚合产物中 DPE 单元在分子链中的键接方式

Scheme 1 The structures of DPE units incorporated in polymer chains 
共聚合), 见图式 2. 因此, AMS 共聚物实际上是一个 “活” 的聚合物(含潜在自由基), 由此可开辟一条新的高 分子合成和改性的路线. 相对于 DPE, AMS 不仅共聚活 性高, 可导致共聚物中的活性点数量较多; 其自由基活 性也相对较高, 有利于后续反应和聚合.

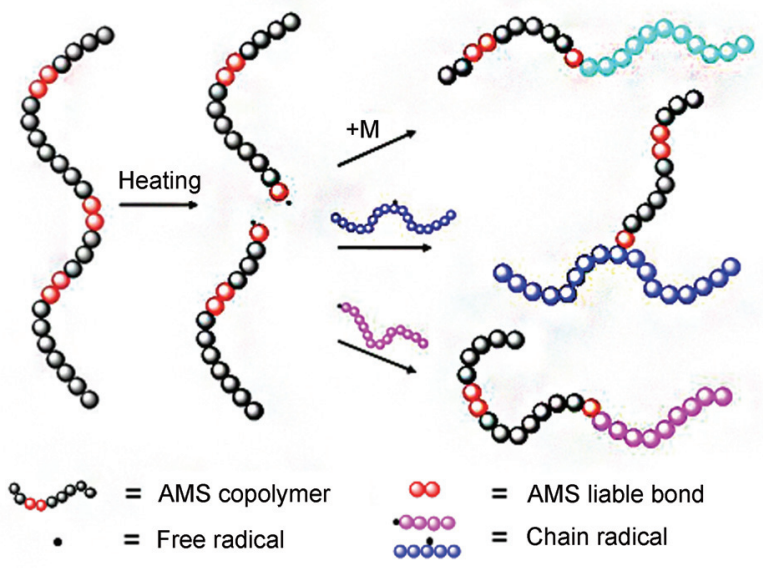

图式 2 AMS 共聚物在高分子合成中的应用

Scheme 2 The application of AMS copolymer as a macromolecular initiator in polymer synthesis

基于此，我们设计合成了一系列 AMS 的共聚物， 然后对其引发自由基聚合的行为进行了评价. 发现这些 共聚物可非常有效地引发各种乙烯基单体的自由基聚 合, 得到各种功能共聚物. 而当无单体存在时, 生成的 链自由基可对聚合物或不饱和碳材料进行直接接枝改 性 ${ }^{[23 \sim 25]}$. 本文将对 “活的” AMS 共聚物的设计合成, 以 及在制备嵌段共聚物、功能化聚合物粒子和大分子改性 领域的反应机理和应用进行介绍.

\section{AMS 共聚物合成}

图 1 是在相同条件下, 甲基丙烯酸缩水甘油酯 (GMA)均聚合和与 AMS 共聚合的转化率与反应时间的 关系 ${ }^{[26]}$. GMA 的均聚合符合典型的自由基聚合规律. 加 入 AMS 后, 单体转化率与反应时间近似成线性增加, 反应 $8 \mathrm{~h}$ 接近 $40 \%(w)$. 单体组成比 AMS/GMA 对共聚 物的组成、 $\overline{M_{\mathrm{n}}}$ 和 $\overline{M_{\mathrm{w}}} / \overline{M_{\mathrm{n}}}$ 的影响见表 $1^{[27]}$. 在 AMS 与 GMA 共聚物 PAG 中, AMS 结构单元的含量可高达约 $25 \%(\mathrm{~mol} \%)$. 而对于 DPE 的调控的聚合体系, 聚合产物
中 DPE 的结构单元的含量低于 $1 \%(\mathrm{~mol} \%)$, 且不随单体 组成中 DPE 量的增加而增大. PAG 的 $\overline{M_{\mathrm{n}}}$ 和 $\overline{M_{\mathrm{w}}} / \overline{M_{\mathrm{n}}}$ 随 着单体中 AMS 的增加而降低, 聚合产物的 $\overline{M_{\mathrm{w}}} / \overline{M_{\mathrm{n}}}$ 远比 GMA 均聚产物的 $\overline{M_{\mathrm{w}}} / \overline{M_{\mathrm{n}}}$ 要小 ${ }^{[23]}$. 单体组成中 AMS 的 含量对共聚合的聚合反应动力学特征、聚合反应产物的 相对分子量及其分布的影响规律，与 DPE 调控的自由 基聚合有相似性 ${ }^{[13]}$ ，但是在共聚物中 AMS 的含量远高 于 DPE.

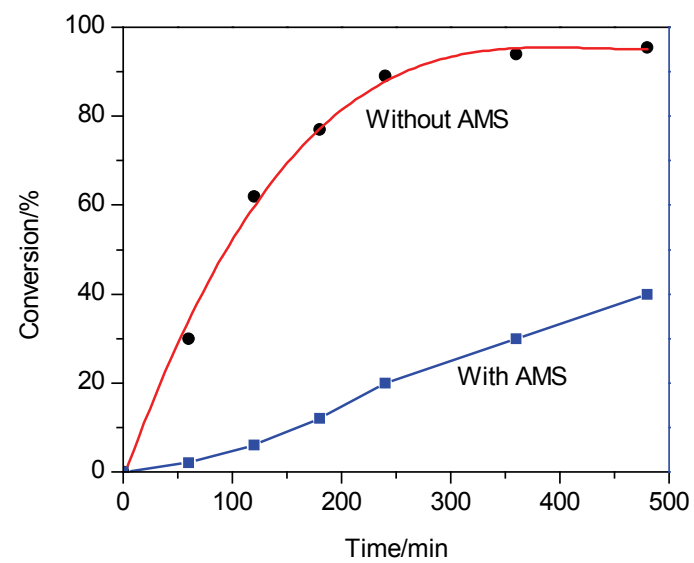

图 1 GMA 与 AMS 共聚合的单体转化率与反应时间的关系

Figure 1 Dependence of monomer conversion as function of time in the polymerization of GMA with AMS

AMS $/ \mathrm{GMA}=1 / 2(\mathrm{~mol} / \mathrm{mol}, \mathrm{AIBN} /(\mathrm{AMS}+\mathrm{GMA})=4 \%(w / w)$ and without AMS (AIBN/GMA $=4 \% w / w)$. The polymerization temperature was $65{ }^{\circ} \mathrm{C}$

聚合产物 PAG 的 ${ }^{1} \mathrm{H}$ NMR 分析表明, 除了 $\mathrm{AMS}$ 与 增长链的加成反应外, 在单体组成中 AMS 含量较高时 $(1 / 3, \mathrm{~mol} \%)$, 终止反应会在共聚物中形成四种键接形式 (图式 3). 前三种来自于 $P_{n}-\mathrm{AMS}_{1}$ 的耦合或者歧化终止 反应, 最后一种来自于 $P_{n}-\mathrm{AMS}_{1}$ 对 $\mathrm{GMA}$ 的加成或与 $P_{n}-\mathrm{GMA}_{1}^{*}$ 的偶合终止. 这些表明由增长链自由基 $P_{n}^{*}$ 与 $\mathrm{AMS}$ 反应生成的链自由基 $P_{n}-\mathrm{AMS}_{1}^{\circ}$ 的反应活性尽管低 于 $P_{n}^{\cdot}$, 却完全不同于 DPE 调控体系(一个大分子链中仅 能结合一个含 DPE 单元的引发点 $)^{[26]}$.

除 GMA 外, AMS 还可以与其它的丙烯酸酯类单体 及苯乙烯共聚合, 得到有引发活性的 AMS 共聚物, 而 且在共聚物中还可以引入马来酸酐 $(\mathrm{MAH})$ 和丙烯酸 (AA)等反应性单体. 表 2 列出的是几个典型的 AMS 共 聚体系结果. 可见, 对于高活性单体, 如 BA, AA 以及

表 1 AMS 和 GMA 共聚合体系单体组成比对聚合反应的影响 ${ }^{a}$

Table 1 Effects of monomer composition on copolymerization of AMS and GMA ${ }^{a}$

\begin{tabular}{|c|c|c|c|c|c|}
\hline \multirow{2}{*}[\mathrm{AMS}]{$/[\mathrm{GMA}](\mathrm{mol} / \mathrm{mol})$} & \multirow{2}{*}{ Yield/\% } & \multicolumn{4}{|c|}{ PAG } \\
\hline & & $\overline{M_{\mathrm{n}}} \times 10^{-3}$ & $\overline{M_{\mathrm{w}}} \times 10^{-3}$ & $\overline{M_{\mathrm{w}}} / \overline{M_{\mathrm{n}}}$ & AMS content $\mathrm{t}^{b} / \mathrm{mol} \%$ \\
\hline $1 / 2$ & 1.61 & 4.57 & 6.19 & 1.31 & 25.6 \\
\hline $1 / 5$ & 2.75 & 5.71 & 8.12 & 1.42 & 11 \\
\hline $1 / 10$ & 11.07 & 12.18 & 19.28 & 1.57 & 7.42 \\
\hline $1 / 20$ & 20.23 & 21 & 36.87 & 1.63 & 3.56 \\
\hline
\end{tabular}

${ }^{a}[\mathrm{AIBN}]=0.1 \%(w), T=65{ }^{\circ} \mathrm{C}, t=7.5 \mathrm{~h},[\mathrm{AMS}]+[\mathrm{GMA}]=25 \%(w) ;{ }^{b}$ Values measured by ${ }^{1} \mathrm{H}$ NMR, referenced to TMS. 


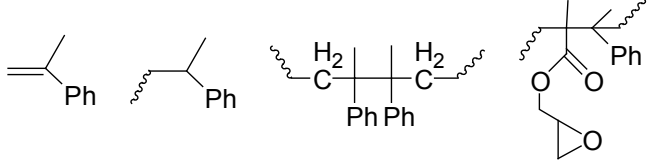

图式 3 AMS 单体单元在共聚物 PAG 分子链中的可能的键接形式 Scheme 3 Possible structures of most AMS units in the PAG

$\mathrm{St} / \mathrm{MAH}$ 等共聚体系，当 $\mathrm{AMS}$ 在单体中的含量为 5\% $10 \%(\mathrm{~mol} \%)$ 时，聚合反应的转化率可以达到 75\% 90\% $(w)$; 其共聚产物中 AMS 单元的含量和共聚物相对分子 质量可以通过 AMS 在单体中的摩尔分数进行调控. 本 体聚合、溶液聚合和乳液聚合均可用来实施 AMS 共聚 物合成.

\section{AMS 共聚物引发自由基聚合的性能}

将 PAG $\left(\overline{M_{\mathrm{n}}}=2900, \overline{M_{\mathrm{w}}} / \overline{M_{\mathrm{n}}}=1.37\right)$ 与 $\mathrm{MMA}$ 在 $90{ }^{\circ} \mathrm{C}$ 加热, 单体转化率与反应时间的关系, 以及聚合 反应产物的 GPC 谱图如图 2 所示. 在大约 $1.5 \mathrm{~h}, \mathrm{MMA}$ 的转化率达 $15 \%(w)$, 未加 PAG 的对照实验没有发生聚 合反应, 说明 MMA 的聚合是由 PAG 引发的. 产物的相 对分子质量远高于作为大分子引发剂的 PAG 的相对分 子质量. 随着反应时间的延长, PAG 的残留峰的相对峰 高变小. 在相同的反应时间, PAG 的 AMS 结构单元含量 越高, 引发的聚合反应单体的转化率也越高 ${ }^{[26]}$.

MMA 在丁酮和甲苯中的溶液聚合实验显示, 反应 温度对 PAG 的引发活性有很大影响. $70{ }^{\circ} \mathrm{C}$ 时 $8 \mathrm{~h}$ 末发现 MMA 的聚合. 而在 $90{ }^{\circ} \mathrm{C}$ 下 $8 \mathrm{~h}$, MMA 的转化率可以达 到 59.6\% (w). AMS 共聚物分解生成自由基有一个临界 反应温度, 温度越高, PAG 的分解反应越快. PAG 的溶 液在 $110{ }^{\circ} \mathrm{C}$ 和 $140{ }^{\circ} \mathrm{C}$ 的热失重也支持了这一结果. 单 体浓度不同时, 相同时间反应的转化率和相对分子质量 也不同. 如单体 MMA 浓度分别为 $0.994 \mathrm{~mol} / \mathrm{L}$ 和 3.776 $\mathrm{mol} / \mathrm{L}$ 时, 反应 $5 \mathrm{~h}$ 后, MMA 的转化率分别为 $20.7 \%(w)$ 和 $66.5 \%(w)$, 相应的 $\overline{M_{\mathrm{n}}}$ 分别为 23200 和 60100 .
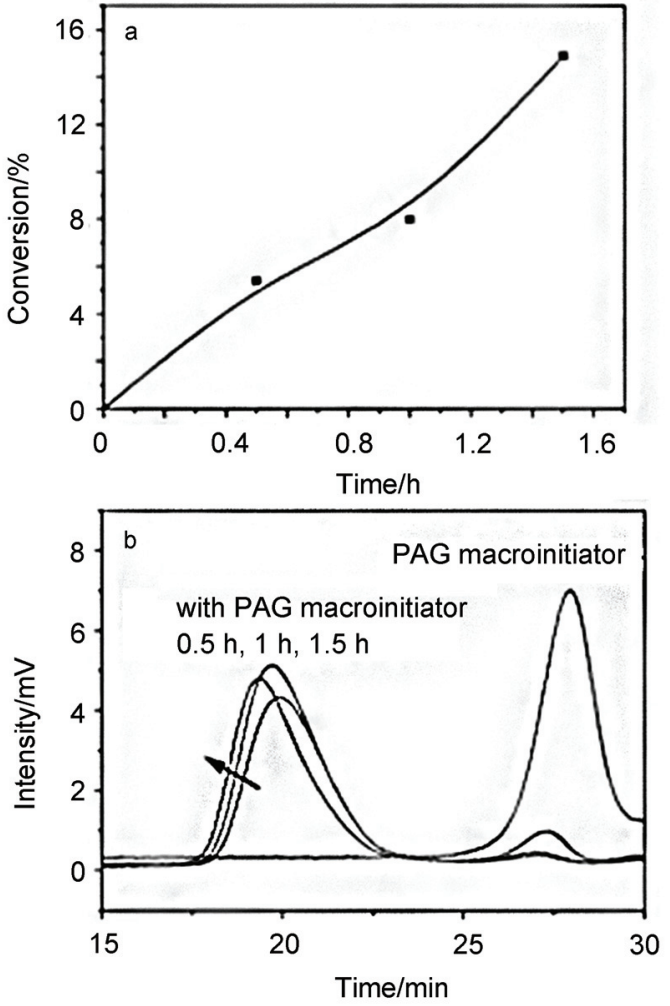

图 2 PAG 共聚物引发的 MMA 本体聚合实验

Figure 2 The bulk polymerization of MMA initiated by PAG precursor $\left(\mathrm{PAG} / \mathrm{MMA}=1 / 2800, \mathrm{~mol} / \mathrm{mol}, T=90{ }^{\circ} \mathrm{C}\right.$ ). (a) Dependence of monomer conversion as function of time; (b) GPC traces of PAG precursor and samples at different time

图 3 是在不同的 PAG 加热时间加入单体 MMA 后, 聚合转化率与时间的关系. 在开始就加入 MMA 的转化 率显著高于在 PAG 加热 $3 \mathrm{~h}$ 后加入 MMA 的实验. 这说 明 PAG 的引发过程是不可逆的，一旦 PAG 裂解生成大 分子链自由基，就会通过解聚和终止反应生成单体和死 的聚合物链. 以苯乙烯为单体的聚合得到了相似的结 果 ${ }^{[25]}$. 这些规律与含 DPE 的大分子引发剂相似 ${ }^{[13,19]}$.

将 1,1-二苯基苦肼基自由基(DPPH)与 $\mathrm{PAG}$ 在 $\mathrm{Ar}$ 保护下于 $90{ }^{\circ} \mathrm{C}$ 反应 $24 \mathrm{~h}$, 产物的 ${ }^{1} \mathrm{H}$ NMR 观察到了 $\mathrm{DPPH}$ 与 $P_{n}-\mathrm{AMS}_{1}^{*}$ 和 $P_{n}-\mathrm{GMA}_{1}$ 自由基的反应产物. 进一

表 2 已研究过的 AMS 共聚合体系汇总

Table 2 The summary of preparing AMS copolymers

\begin{tabular}{|c|c|c|}
\hline Monomers and polymerization system & Experimental results & Reference \\
\hline GMA/AMS, in THF with AIBN & $\begin{array}{l}f_{\mathrm{AMS}}=1 / 2,1 / 9,0.5 / 9.5 ; F_{\mathrm{AMS}}=0.225,0.078,0.025 ; M_{\mathrm{n}}=3100,4500,6500 ; M_{\mathrm{w}} / M_{\mathrm{n}} \\
=1.26,1.45,1.48\end{array}$ & {$[26]$} \\
\hline AMS/BA/AA, Soap-free emulsion with KPS & {$[\mathrm{AMS}] /[\mathrm{BA}]=2 / 1,1 / 1 ; 1 / 2,1 / 10 ; M_{\mathrm{n}}=12900,13600,31300,57400$} & [28] \\
\hline AMS/AA/MMA, emulsifier-free emulsion with KPS & $\begin{array}{l}{[\mathrm{AA}] /[\mathrm{AMS}]+[\mathrm{MMA}]=1 / 3, f_{\mathrm{AMS}}=0.083 \sim 0.25(\mathrm{AMS} / \mathrm{MMA}) ; \text { Yield }=69 \% \sim} \\
90 \%(w) ; M_{\mathrm{n}}=96000 \sim 29000 ; M_{\mathrm{w}} / M_{\mathrm{n}}=3.2 \sim 2.5\end{array}$ & [29] \\
\hline $\mathrm{AMS} / \mathrm{St} / \mathrm{MAH}$, in Butanone with AIBN & $\begin{array}{l}{[\mathrm{AMS}] /[\mathrm{St}+\mathrm{MAH}]=1 / 1,1 / 2,1 / 3,1 / 4,1 / 5 ; \text { Yield }=20.5 \%, 37.3 \%, 45.3 \%, 67.4 \%,} \\
75.7 \%(w) ; M_{\mathrm{n}}=9250,9570,8130,9880,16120 ; M_{\mathrm{w}} / M_{\mathrm{n}}=1.79,2.39,3.21,2.75, \\
2.48 .\end{array}$ & {$[30]$} \\
\hline
\end{tabular}

BA: butyl acrylate, AA: acrylic acid; MAH: maleic anhydride, THF: tetrahydrofuran. $f_{\text {AMs }}$ : molar fraction of AMS in monomers; $F_{\text {AMS: }}$ molar fraction of AMS unit in copolymers. 


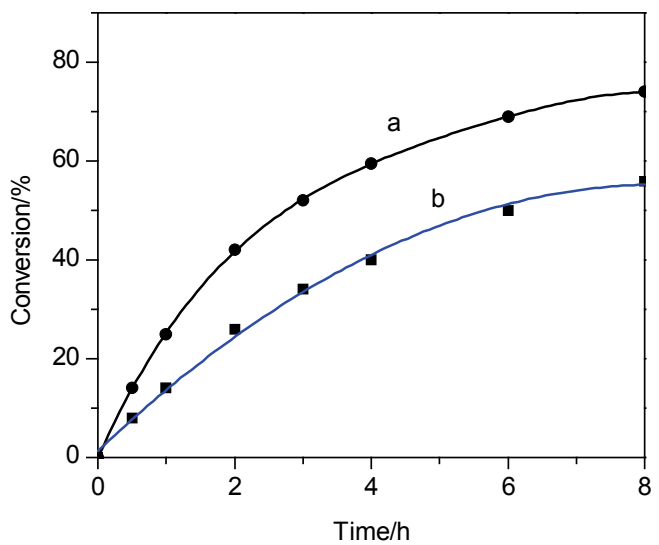

图 3 PAG 引发的 MMA 溶液聚合单体转化率与 MMA 加入时间的关 系

Figure 3 Time dependence of monomer conversion with MMA added at different time

(a) adding MMA at the beginning of PAG degradation and (b) adding MMA after $3 \mathrm{~h}$ PAG degradation at $110{ }^{\circ} \mathrm{C}(\mathrm{PAG} / \mathrm{MMA} /$ toluene $=1 \mathrm{~g} / 10 \mathrm{~mL} / 50$ $\mathrm{mL}$, reaction time $8 \mathrm{~h}, T=110{ }^{\circ} \mathrm{C}$ ).

步，将 $N$-叔丁基- $\alpha$-苯基硝酩(PBN)与 PAG 溶于甲苯中, 在 $\mathrm{Ar}$ 的保护下于 $90{ }^{\circ} \mathrm{C}$ 加热 $30 \mathrm{~min}$, 产物的 $\mathrm{ESR}$ 超精 细偶合常数为 $\alpha^{H}=2.67, \alpha^{N}=14.35$, 表明生成的为 $\mathrm{C}$ 中 心自由基. 上述事实表明 AMS 的共聚物在加热时能分 解产生自由基 ${ }^{[28]}$.

AMS 与 MAH 的共聚物 Poly(AMS-co-MAH)在加热 时也能引发 MMA 的聚合. 图 4 为 $90{ }^{\circ} \mathrm{C}$ 下聚合反应 12 $\mathrm{h}$, MMA 转化率和产物相对分子质量与 Poly(AMS$c o$-MAH)用量的关系. 随 Poly(AMS-co-MAH)用量的增 加, MMA 的转化率增大, 产物的相对分子质量降低, 20 $\mathrm{h}$ 的转化率可以达到 $75 \%(w)$ 以上. 这一规律与小分子 引发剂的结果相似, 说明 Poly(AMS-co-MAH)起到了引 发作用. 以苯乙烯为单体, 用 Poly(AMS-co-MAH)引发 的聚合也得到了相似的结果 ${ }^{[31]}$.

AMS 共聚物的引发机理与常规自由基引发剂相似, AMS 共聚物分解产生大分子链自由基的过程是一个慢 速反应, 大分子链自由基引发聚合的链增长反应是快速 反应(传统自由基聚合), 聚合的终止反应与传统自由基 聚合一样与单体的性质和反应体系有关. AMS 共聚物引 发的聚合有独特之处: (1) AMS 共聚物引发的聚合可以 得到嵌段共聚物, 而且根据需要可以对嵌段进行功能设 计(如引入羧基、酸䣲基和其它基团); (2)产物不含有小 分子引发剂的残留物; (3) 新生成的聚合物链段不含 AMS 结构单元, 因此聚合反应的链终止是不可逆的.

\section{AMS 共聚物引发乳液聚合}

将 Poly(AMS-co-MAH)用氨水进行㿝化, 可以得到 具有引发剂和乳化剂双重功能的 AMS 共聚物, 用于引 发 MMA 的无皇乳液聚合，单体的转化率可以达到 $85 \%$

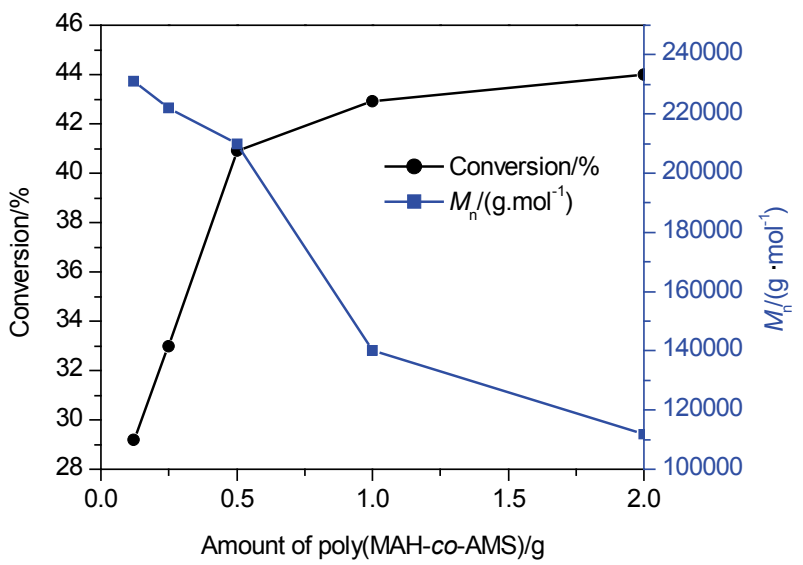

图 4 以 Poly(MAH-co-AMS)为大分子引发剂的 MMA 聚合单体转化 率和聚合物相对分子质量与 MMA/Poly(MAH-co-AMS)比的关系

Figure 4 MMA conversion and molecular weight of PMMA initiated by Poly(MAH-co-AMS) with different MMA/Poly(MAH-co-AMS) ratios solvent: 2-butanone, $90{ }^{\circ} \mathrm{C}, 12 \mathrm{~h}$

$(w)$ ，乳胶粒子的尺寸为 $150 \sim 200 \mathrm{~nm}$. MMA 反应的速 率随大分子引发剂用量的增加而增大, 并且产物的特性 粘数随 MMA 转化率的增加而增大(如图 5 所示). 结构 分析表明, 得到的产物是以 Poly(AMS-co-MAH)为短链 段, PMMA 为长链段的嵌段共聚物 ${ }^{[32]}$.

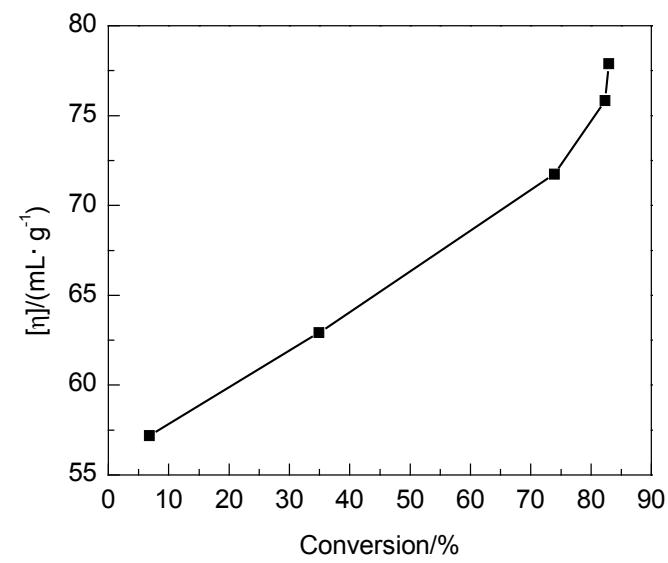

图 5 MMA 无㿝乳液聚合产物特性粘数与单体转化率的关系

Figure 5 The intrinsic viscosity numbers of copolymers against conversion

MMA: $12 \mathrm{~g}$, Macroinitiator: $1.8 \mathrm{~g}, \mathrm{H}_{2} \mathrm{O}: 60 \mathrm{~mL}, T=90{ }^{\circ} \mathrm{C}, t=4 \mathrm{~h}$

AMS 共聚物大分子引发剂也可以用于制备两亲性 的核壳结构聚合物纳米粒子. 以 AMS, BA 和 AA 为单 体, 合成三元共聚物 PABA. 然后以 PABA 为大分子引 发剂, 在 $90{ }^{\circ} \mathrm{C}$ 下引发苯乙烯的无㿝乳液聚合, 可以得 到粒径为 $126 \mathrm{~nm}$ 到 $282 \mathrm{~nm}$ 的具有核壳结构的聚合物粒 子(PABA 为壳, PS 为核), TEM 图像如图 6 所示. IR 和 GPC 谱图显示得到的聚合物为大分子引发剂引发产生 的 ${ }^{[33]}$. 


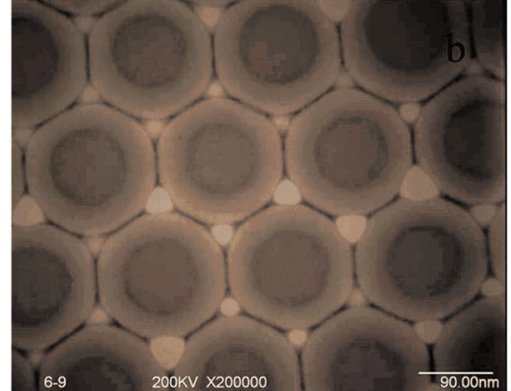

图 6 以 AMS 共聚物为引发剂的核壳结构共聚物粒子的 TEM 图像 Figure 6 TEM pictures of core-shell copolymer particles (scale bar $=90$ $\mathrm{nm})\left(\mathrm{PSt} / \mathrm{PABA}=3: 2(w / w), T=90{ }^{\circ} \mathrm{C}\right)$.

\section{AMS 共聚物的原位接枝改性}

利用含 AMS 的共聚物在受热时易于断链产生自由 基的特性，我们提出了利用 AMS 共聚物熔融接枝增容 的新方法 ${ }^{[23,30,34 ~ 37]}$. 在共混过程中, AMS 齐聚物受热断 链生成自由基, 通过链转移反应接枝到聚合物链上，原 位产生的接枝聚合物为相容剂. 由于共混体系中仅存在 大分子链自由基, 有利于抑制熔融接枝过程的分子链降 解. 而且, 在共混物中也不会有小分子单体和引发剂的 残留，是一种绿色的熔融接枝增容技术.

对 PP/Nylon 66 共混体系, 加入 1 份的 PAG 可以提 高拉伸强度 $12.0 \%$ 、断裂伸长率 $14.4 \%$ 和冲击强度 $14.1 \%$, 明显起到了增韧和增强的效果. 图 7 是不同 PAG 用量时, PP/Nylon 66/PAG 共混物断面的 SEM 图像 (PP 为连续相, Nylon 66 为分散相). 未加入 PAG 的对照 样存在明显的相分离, Nylon 66 微区的大小约为 $2 \sim 20$ $\mu \mathrm{m}$ (图 7a). 加入 5\% (w) 的 PAG, 两相之间的相容性得 到了明显的提高. 随 PAG 中 AMS 含量的增加, 两相界 面作用明显改善(图 $7 b \sim d$ ). 当 $P A G$ 中 $A M S$ 含量达到
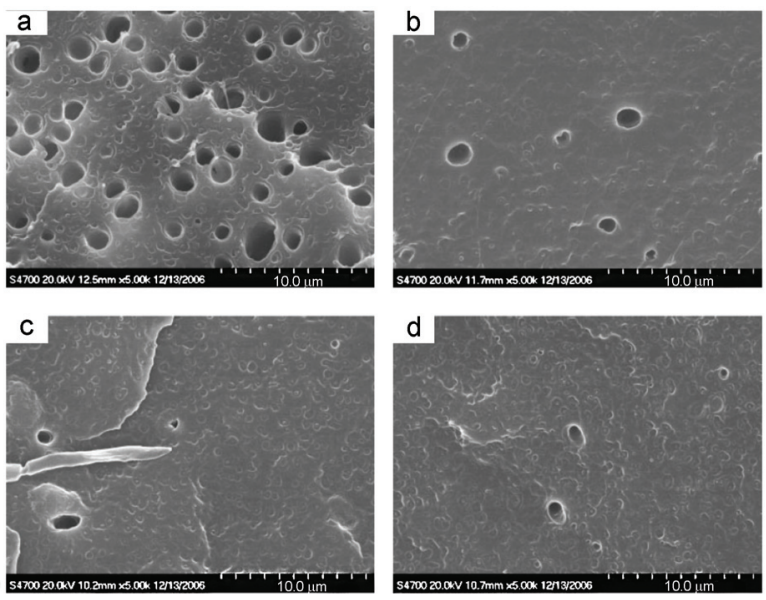

图 $7 \mathrm{PP} /$ Nylon 66 共混物的 SEM 图(标尺为 $100 \mu \mathrm{m}$ )

Figure 7 SEM micrographs of the PP/Nylon (The scale bar is $100 \mu \mathrm{m}$ ). (a) PP/Nylon $66(80 / 20, w / w)$; (b) PP/Nylon 66/PAG $(76 / 19 / 5, w / w / w)$, the AMS content in PAG was $11.0 \mathrm{~mol} \%$; (c) PP/Nylon 66/PAG $(76 / 19 / 5, w / w / w)$, the AMS content in PAG was $16.4 \mathrm{~mol} \%$; and (d) PP/Nylon 66/PAG $(76 / 19 / 5$, $w / w / w)$, the AMS content in PAG was $25.6 \mathrm{~mol} \%$.
11.6\% (mol\%)时, Nylon 66 的微区尺寸降低到 $10 \mu \mathrm{m}$ 以 下. 随着 PAG 中 AMS 含量的进一步增加, PP 和 Nylon 66 的相界面变得不明显(图 7d $)^{[34]}$.

图式 4 是以 PAG 为反应性增容剂, PP 和 Nylon 66 共混的增容机理示意图 ${ }^{[34]}$. PP 和 PAG 在 Brabender 塑化 仪中混炼反应的产物用丙酮抽提后的 IR 谱图中, 出现 了 $1729 \mathrm{~cm}^{-1}$ 的 $\mathrm{C}=\mathrm{O}$ 吸收峰以及 $760 \mathrm{~cm}^{-1}$ 和 $704 \mathrm{~cm}^{-1}$ 处的苯环吸收峰, 证明 $\mathrm{PAG}$ 齐聚物接枝到了 PP 分子链 上.

显然, AMS 共聚物分解生成自由基是原位接枝增容 的关键. PGMA 和 PAG 的热失重曲线见图 8. PGMA 只 有一个分解过程, 转变温度的中值为 $305.7{ }^{\circ} \mathrm{C}$. 而 $\mathrm{PAG}$ 有两个分解区, 分别为 $178.2{ }^{\circ} \mathrm{C}$ 和 $342.1{ }^{\circ} \mathrm{C}^{[26]}$. 这表明 在 PAG 中存在对热敏感的弱键结构, 受热时易断裂. PAG 分子链开始断裂的温度, 低于常用聚合物的熔融 加工温度, 有利于在熔融过程中进行接枝. 更进一步, 随着 PAG 中 AMS 含量的增加, 起始分解温度降低, PAG 分子链中由弱键热分解产生的自由基数量也随之 增加, 热失重量增大. $\mathrm{PAG}$ 升温到 $260{ }^{\circ} \mathrm{C}$ 后的等温热失 重结果显示, 最终的热失重率也随着 AMS 结构单元含 量的增加而增加. 进一步说明 PAG 分子链中的弱键结 构存在于与 AMS 相连的链节中.

苯乙烯, MAH 和 AMS 的三元共聚物 PASM 也可以 在 PP/Nylon 的共混中通过原位接枝起增容作用 ${ }^{[30]}$. 在 PP/Nylon $6(80 / 20, w / w)$ 共混体系中加入 $3 \%(w)$ 的 PASM 就可以有效地降低 PP 和 Nylon 6 的两相界面张 力, 使共混体系中 PP 和 PP/Nylon 6 的相容性得到明显 的改善, 分散相 Nylon 6 的颗粒尺寸由对比试验的 $5 \sim 8$ $\mu \mathrm{m}$ 减小至 $1 \sim 2 \mu \mathrm{m}$.

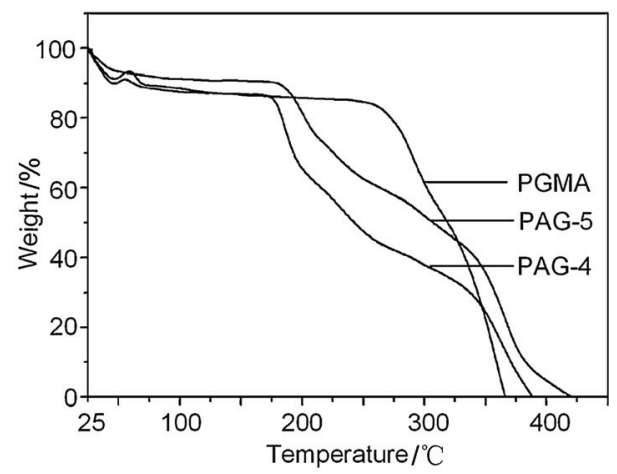

图 8 不同 AMS 含量的 PAG 共聚物及 PGMA 的 TGA 曲线 Figure 8 TGA curves of PAG copolymers and PGMA

PAG-4 $\left(M_{\mathrm{n}}=6200, F_{\mathrm{AMS}}=0.199\right)$ and PAG-5 $\left(M_{\mathrm{n}}=9400, F_{\mathrm{AMS}}=0.038\right)$, temperature range: $25 \sim 450{ }^{\circ} \mathrm{C}$, rate: $10{ }^{\circ} \mathrm{C} / \mathrm{min}, \mathrm{N}_{2}$

研究还发现, AMS 齐聚物还可以用于纳米粒子的接 枝改性. 例如将 PAS 或 PAG 溶于 DMF 或甲苯溶剂中, 加入多壁碳纳米管(MWCNT)超声分散. 然后在 $\mathrm{Ar}$ 气保 护下，于 $90{ }^{\circ} \mathrm{C}$ 加热反应 $24 \mathrm{~h}$, 即可得到 PAS 或 PAG 接

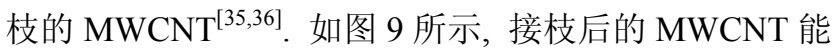




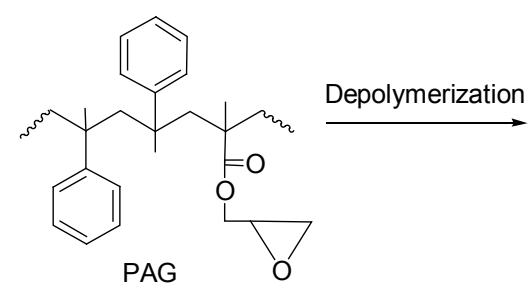<smiles>CCC(C)(CC(C)(CC(C)(CC)c1ccccc1)C(=O)OCC1CO1)c1ccccc1</smiles><smiles>[R17]C=CCCC(C)(C)CC(CC)(CC)c1ccccc1</smiles>

$\mathrm{R}_{1}^{\bullet}$

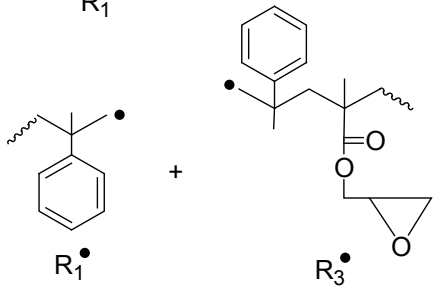<smiles>CC(C)CC(C)CC(C)CC(C)CC(C)C</smiles>

Degradation

PP
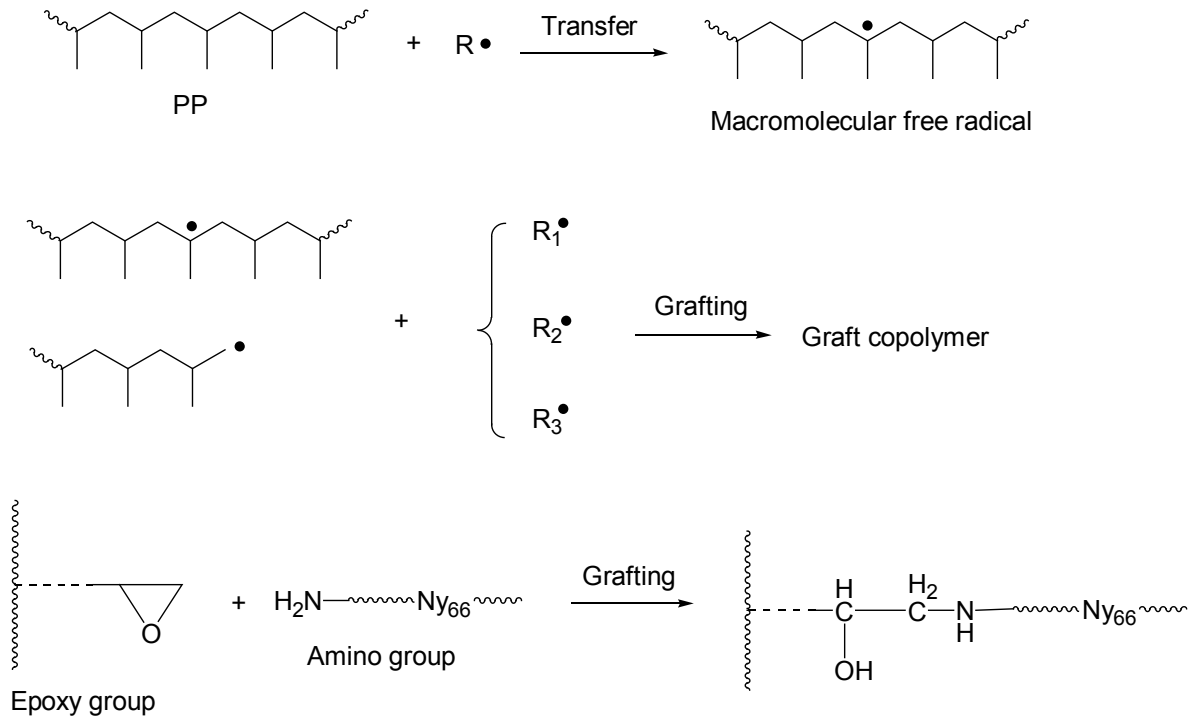

图式 4 PP/Nylon 66/PAG 反应性增容体系的机理示意图

Scheme 4 Schematic representation of a possible mechanism for the reactive compatibilization of PP/Nylon 66

稳定地分散在丙酮和 DMF 中. 产物的拉曼光谱显示, MWCNT 经 PAG 处理后 $I_{D} / I_{G}=0.4$, 而未处理 MWCNT 的 $I_{D} / I_{G}=0.28$, 说明 PAG 分子链与 MWCNT 之间形成 了共价键. 处理后 MWCNT 样品的 IR 谱图和 XPS 谱图 也证实 PAS 和 PAG 与 MWCNT 发生了接枝反应.

\section{6 结论和展望}

提出并实验证明了含 AMS 结构单元的共聚物是一 种 “活” 的聚合物概念和方法. 首先, 由这种活的共聚 物代替传统的过氧和偶氮类小分子引发剂, 引发乙烯基 单体的自由基聚合, 可摈除残留引发剂问题, 有绿色特 点. 其次, 通过对共聚物中不同功能单体的选择, 可制
备多种嵌段或杂链功能共聚物和粒子。最后，结合反应 性基团(如环氧, 酐基等)选择引入, 这些活的共聚物不 仅可对多相体系进行原位接枝增容，还可对无机粒子和 不饱和的碳纳米材料进行原位表面改性.

这种新的高分子合成和改性策略具有极大的工业 应用前景，将来的研究工作应关注两大应用领域. 一是 设计和合成含功能性基团如羧基、酸酐和磷酸根(酯)的 嵌段共聚物, 开发高性能的分散剂和增溶剂. 这方面的 应用已受到了工业界的关注，例如，最近，有公司利用 含 AMS 的共聚物为大分子引发剂, 制备了具有优异分 散性能的两亲性嵌段共聚物分散剂, 并认为这一技术很 好地解决了嵌段共聚物的制备成本问题 ${ }^{[38]}$. 另一个应 


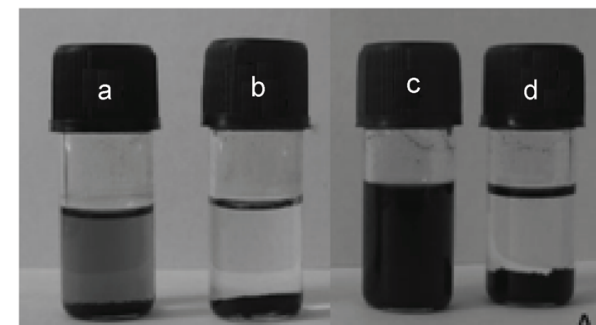

图 $9 \mathrm{AMS}$ 共聚物 PAG 处理前后 MWCNT 在丙酮和 $N, N$-二甲基甲酰 胺中的分散情况

Figure 9 Dispersion of MWCNT in acetone and DMF before and after heated with AMS copolymer PAG

(a) PAG- $g$-MWCNTs in acetone, (b) raw MWCNTs in acetone, (c) PAG-g-MWCNTs in DMF, and (d) raw MWCNTs in DMF

用领域是设计合成含不同官能团的 AMS 齐聚物，应用 于聚合物的共混和填充体系, 利用 AMS 共聚物的熔融 接枝反应，实现原位增容和分散，开发高性能、无小分 子残留的绿色共混和填充聚合物材料.

\section{作者简介}

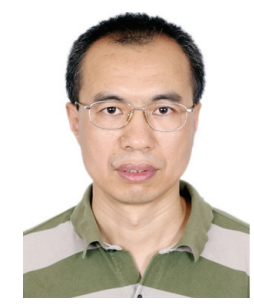

马育红, 1966 年出生, 博士, 北京化工大学材料科学与工 程学院副教授, 博士生导师. 1987 年在北京化工学院高分子系 获得学士学位, 1990 年在北京化工学院高分子系获得硕士学 位. 2003 年在北京化工大学材料科学与工程学院获得博士学 位，导师杨万泰教授. 2004 年至 2008 年在基美电子（苏州） 有限公司工作. 2008 年至今在北京化工大学材料科学与工程 学院工作. 主要研究领域为自由基光聚合、阳离子聚合、导电 聚合物及器件等.

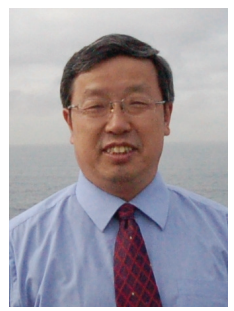

杨万泰, 1956 年出生, 博士, 北京化工大学材料科学与工 程学院教授, 院长. 1982 年在清华大学化工系获得学士学位, 1985 年在北京化工学院高分子系获得硕士学位. 1996 年在瑞 典皇家工学院 (RIT) 聚合物技术系获得博士学位, 导师 Bengt Rånby 教授. 1985 年起在北京化工大学高分子系任教, 1996 年 任教授, 2000 年受聘教育部 “长江学者” 计划特聘教授, 2001 年获得国家杰出青年基金. 2006 年起当选中国感光学会副理 事长. 主要研究领域为有机材料表面化学、活性自由基聚合、 聚合反应新方法等.

\section{References}

[1] Moad, G.; Solomon, D. H. The Chemistry of Radical Polymerization, 2nd ed., Elsevier, Oxford, UK, 2006, pp. 1 9.

[2] Greszta, D.; Mardare, D.; Matyjaszewski, K. Macromolecules 1994, 27,638 .

[3] Wade, A. B.; Matyjaszewski, K. Prog. Polym. Sci. 2007, 32, 93.

[4] Otsu, T.; Yoshida, M. Makromol. Chem. Rapid Commun. 1982, 3, 127.

[5] Otsu, T. J. Polym. Sci., Part A: Polym. Chem. 2000, 38, 2121.

[6] Solomon, D. H.; Rizzardo, E.; Cacioli, P. US Patent 4581429, 1985 [Chem. Abstr. 1985, 102, 221335].

[7] Wang, J. S.; Matyjaszewski, K. J. Am. Chem. Soc. 1995, 117, 5614.

[8] Kato, M.; Kamogato, M.; Sawamoto, M.; Higashimura, T. Macromolecules 1995, 28, 1721 .

[9] Percec, V.; Barboiu, B. Macromolecules 1995, 28, 7970.

[10] Chiefari, J.; Chong, K.; Ercole, F; Krstina, J.; Jeffery, J.; Le, T. P. T.; Mayadunne, R. T. A.; Meijs, G. F.; Moad, C. L.; Moad, G.; Rizzardo, E.; Thang, S. H. Macromolecules 1998, 31, 5559.

[11] Higashihara, T.; Hayashi, M.; Hirao, A. Prog. Polym. Sci. 2011, 36, 323.

[12] Kwon, Y.; Faust, R.; Chen, C. X.; Thomas, E. L. Macromolecules 2002, 35, 3348.

[13] Wieland, P. C.; Raether, B.; Nuyken, O. Macromol. Rapid Commun. 2001, 22, 700 .

[14] Kos, T.; Strissel, C.; Yagci, Y.; Nugay, T.; Nuyken, O. Eur. Polym. J. 2005, 41, 1265.

[15] Luo, Y.-D.; Chou, I.-C.; Chiu, W.-Y.; Lee, C.-F. J. Polym. Sci.: Part A: Polym. Chem. 2009, 47, 4435.

[16] Chen, D.; Shi, Y.; Fu, Z.-F. J. Appl. Polym. Sci. 2009, 111, 1581.

[17] Guo, F. G.; Zhang, Q. Y.; Luo, S. B.; Zhang, H. P. Acta Polym. Sinica 2010, (5), 508.

[18] Wang, W. W.; Zhang, Q. Y. J. Colloid Interface Sci. 2012, 374, 54.

[19] Raether, B.; Nuyken, O.; Wieland, P. C.; Bremser, W. Macromol. Symp. 2002, 177, 25.

[20] Lowry, G. G. J. Polym. Sci. 1960, 42, 463.

[21] Worsfold, D. J.; Bywater, S. J. Polym. Sci. 1957, 26, 299.

[22] Madras, G.; Smith, J. M.; McCoy, B. J. Polym. Degrad. Stab. 1996 $52,349$.

[23] Yang, W.-T.; Jiang, S.; Liang, S.-J.; Deng, J.-P.; Ma, Y.-H.; Sun, Y.-F.; Liu, L.-Y.; He, C.-F. CN 200610012074, 2006 [Chem. Abstr. 2007, 146, 163609].

[24] Liang, S.-J. Ph.D. Dissertation, Beijing University of Chemical Technology, Beijing, 2007. (梁淑君, 博士论文, 北京化工大学, 北京, 2007.)

[25] Jiang, S. Ph.D. Dissertation, Beijing University of Chemical Technology, Beijing, 2009. (蒋姗, 博士论文, 北京化工大学, 北京, 2009.)

[26] Jiang, S.; Deng, J.-P.; Yang, W.-T. Polym. J. 2008, 40, 543.

[27] Liang, S.-J.; Deng, J.-P.; Yang, W.-T. Chin. J. Polym. Sci. 2010, 28, 323.

[28] Jiang, S.; Deng, J.-P.; Yu, Q.; Yang, W.-T. J. Appl. Polym. Sci. 2011, $120,466$.

[29] Jiang, S.; Sun, A.-P.; Deng, J.-P.; Yang, W.-T; Yu, Q. J. Macromol. Sci. Part A: Pure and Appl. Chem. 2011, 48, 846.

[30] Liang, S.-J.; Yang, W.-T. Acta Polym. Sinica 2011, (2), 180. (梁淑 君, 杨万泰, 高分子学报, 2011, (2), 180.)

[31] Chen, C. M.S. Thesis, Beijing University of Chemical Technology, Beijing, 2011. (陈铖, 硕士论文, 北京化工大学, 北京, 2009.)

[32] Cao, L.-J.; Yin, M.-Z.; Ma, Y.-H.; Yang, W.-T. Acta Polym. Sinica 2012, (1), 75. (曹丽娟, 尹梅贞，马育红，杨万泰，高分子学报， 2012, (1), 75.)

[33] Jiang, S.; Fang, X.-H.; Yu, Q.; Deng, J.-P.; Yang, W.-T. J. Appl. Polym. Sci. 2012, 124, 4121.

[34] Deng, J.-P.; Liang, S.-J.; Zhang, C.-R.; Yang, W.-T. Macromol. Rapid Commun. 2007, 28, 2163.

[35] Liang, S.-J.; Yang, W.-T. e-Polymers 2010, no. 092.

[36] Jiang, S.; Deng, J.-P.; Yang, W.-T. Macromol. Rapid Commun. 2008, 29, 1521.

[37] Liang, S.-J.; Yang, W.-T. Chin. Plast. 2010, 24, 34. (梁淑君，杨万 泰, 中国塑料, 2010, 24, 34.)

[38] Cui, L.-L.; Feng, S.-G.; Liu, Y.-F.; Wang, T.; Zhang, Q.-W. US 20120101218A1, 2012 [Chem. Abstr. 2012, 156, 534374].

(Cheng, B.; Lu, Z.) 